\title{
Spontaneous coronary artery dissection: from expert consensus statements to evidence-based medicine
}

\author{
Fernando Alfonso, Marcos García-Guimaraes, Teresa Bastante, Francisco de la Cuerda, Paula Antuña, \\ Javier Cuesta, Fernando Rivero \\ Department of Cardiology, Hospital Universitario de La Princesa, Madrid, Spain \\ Contributions: (I) Conception and design: F Alfonso; (II) Administrative support: F Alfonso; (III) Provision of study materials or patients: All authors; \\ (IV) Collection and assembly of data: All authors; (V) Data analysis and interpretation: All authors; (VI) Manuscript writing: All authors; (VII) Final \\ approval of manuscript: All authors. \\ Correspondence to: Fernando Alfonso, MD. Department of Cardiology, Hospital Universitario de La Princesa, Instituto de Investigación Sanitaria \\ Princesa (IIS-IP), Universidad Autónoma de Madrid, Diego de León 62, Madrid 28006, Spain. Email: falf@hotmail.com.
}

\begin{abstract}
Spontaneous coronary artery dissection (SCAD) represents an increasingly recognized cause of acute coronary syndrome (ACS), especially in young females. Novel diagnostic insights obtained from intracoronary imaging and the frequent association with fibromuscular dysplasia (FMD), have recently enriched our understanding of this unique clinical entity. Recently, two scientific statements from both sides of the Atlantic, summarizing the available information on SCAD, have been simultaneously published. These should help to guide the clinical management of these challenging patients. Major collaborative research efforts are required to move from expert-based recommendations to evidence-base medicine.
\end{abstract}

Keywords: Spontaneous coronary artery dissection (SCAD); intramural hematoma; optical coherence tomography (OCT); intravascular ultrasound; fibromuscular dysplasia (FMD); myocardial infarction

Submitted Jun 20, 2018. Accepted for publication Jun 29, 2018.

doi: $10.21037 /$ jtd.2018.07.10

View this article at: http://dx.doi.org/10.21037/jtd.2018.07.10

Spontaneous coronary artery dissection (SCAD) is emerging as an important cause of acute coronary syndrome (ACS), particularly in young females (1-3). Heightened awareness and novel diagnostic tools, especially intracoronary imaging, suggest that $\mathrm{SCAD}$ is much more prevalent than previously thought (1-3). Classical information, stemming for a myriad of single-case reports and many small retrospective case series, has been recently enriched with data from relatively large series of patients from different countries (1-3). Main differences from atherosclerotic coronary artery disease (ACAD) are now well established. Triggers and concurrent systemic conditions have been extensively studied. Recently, association with fibromuscular dysplasia (FMD) and other arteriopathies, has been the focus of intense research. Currently, management strategies, medical therapy and revascularization recommendations significantly differ from those used in patients with ACS as a result of ACAD (1-3).

Early this year two different scientific statements on
SCAD from both sides of the Atlantic [American Heart Association (AHA) and European Society of Cardiology (ESC)], were simultaneously published $(1,2)$. These systematic and comprehensive consensus documents were generated from experts in the field after critically reviewing all the available information $(1,2)$. These multicenter consensuses represent a major step forward from previous review papers on this clinical entity $(3,4)$. In these scientific statements current knowledge on SCAD was nicely summarized but management recommendations were still cautious, limited, and mostly based in experts' opinion $(1,2)$.

In this paper we will review the main issues addressed in the AHA scientific statement (1).

\section{Definition, epidemiology and pathophysiology}

SCAD is defined as an epicardial coronary artery dissection that is not associated with ACAD or trauma. Therefore, 


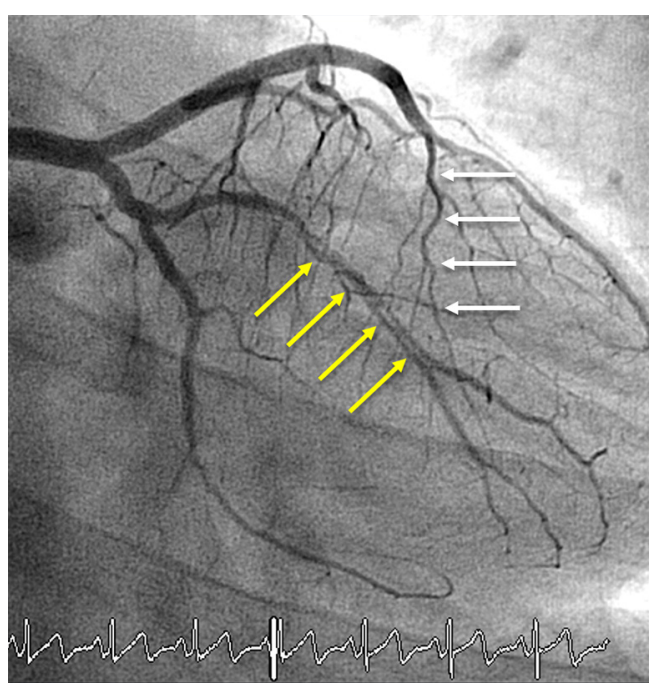

Figure 1 Coronary angiogram of a patient with SCAD revealing a double lumen with a clear dissection flap at the first marginal branch (yellow arrows). In addition, a sudden diffuse narrowing of the distal left anterior descending coronary artery (white arrows) is detected after the take-off of a large diagonal branch. SCAD, spontaneous coronary artery dissection.

patients with iatrogenic dissections and those with associated ACAD (included in some previous series) are not considered in the AHA statement. Intimal disruption or intramural hematoma represent the pathologic substrate of SCAD. True prevalence remains unknown, as the condition frequently remains underrecognized. However, it has been estimated that SCAD accounts for 1-4\% of all ACS patients (1). Importantly, SCAD may constitute the underlying etiology of myocardial infarction in up to onethird of women aged $<50$ years $(1,5)$. Average age at diagnosis in different series ranges from 45 to 55 years old. The vast majority of patients are women with a low prevalence of risk factors. The left anterior descending coronary artery is the most frequently involved vessel but $10-20 \%$ of patients present with multivessel involvement (1).

The two classical theories explaining the formation of a false lumen include intimal disruption (intimal tear) and intramural hematoma from leaking vasa vasorum. Periadventitial inflammation with eosinophil predominance is frequently found. The fundamental cause of SCAD remains unknown but currently it is considered to be multifactorial (1).

The association with systemic arteriopathies and FMD in particular (20-85\% of cases) has been well-established
$(1,6)$. FMD is a noninflammatory, nonatherosclerotic, vascular entity that manifests with stenosis, aneurysms, tortuosity or dissections. A multifocal morphology, typically providing the "string-of-beds" pattern, is the most common type of FMD. FMD most frequently affects renal, iliac and extracranial carotid arteries. The presence of "coronary" FMD has been also recently reported (7).

The term "pregnancy-associated" SCAD rather than peripartum SCAD, is currently preferred (1). Prevalence has been reported as 1.8 SCAD events every 100,000 pregnancies (1). Hormonal-related changes in the artery wall architecture have been implicated. Preeclampsia seems to be a risk factor. Notably, pregnancy-associated SCAD appears to convey a particularly poor prognosis (1).

Despite description in classical reports, association with systemic inflammatory (lupus erythematosus, inflammatory bowel disease, sarcoidosis, polyarteritis nodosa) or connective tissue disorders (Ehlers-Danlos, Marfan, LoeysDietz, polycystic kidney) is rare (1).

Many potential precipitants and triggers have been described. Emotional stressors are more frequent in women whereas physical stressors (isometric exercise, weight lifting) are more common in men. Catecholamine surges increasing coronary shear stress have been implicated. Contraceptives and postmenopausal hormone therapy have also been suggested as potential triggers of SCAD (1).

\section{Clinical and diagnostic issues}

Clinical presentation is similar to that seen in patients with ACAD. Most patients present as ACS, frequently as STsegment elevation myocardial infarction (1). Cardiac enzymes are universally increased. Presentation as cardiogenic shock, ventricular arrhythmias or sudden death, is rare (1).

Despite increasing awareness, SCAD continues to be underdiagnosed or misdiagnosed as ACAD. However, precise early diagnosis is considered of paramount importance as management differs from that in patients with ACS secondary to ACAD.

Coronary angiography, despite its inherent limitations, remains the first-line diagnostic tool. This technique is widely available and, therefore, instrumental for the initial diagnosis. The pathognomonic image of a double lumen morphology with contrast staining, or with a clear dissecting flap, is only detected in one-third of patients (1-4) (Figure 1). Accordingly, precise interpretation of subtle angiographic signs remains critical when clinical suspicious is high (i.e., young female without risk factors and smooth 

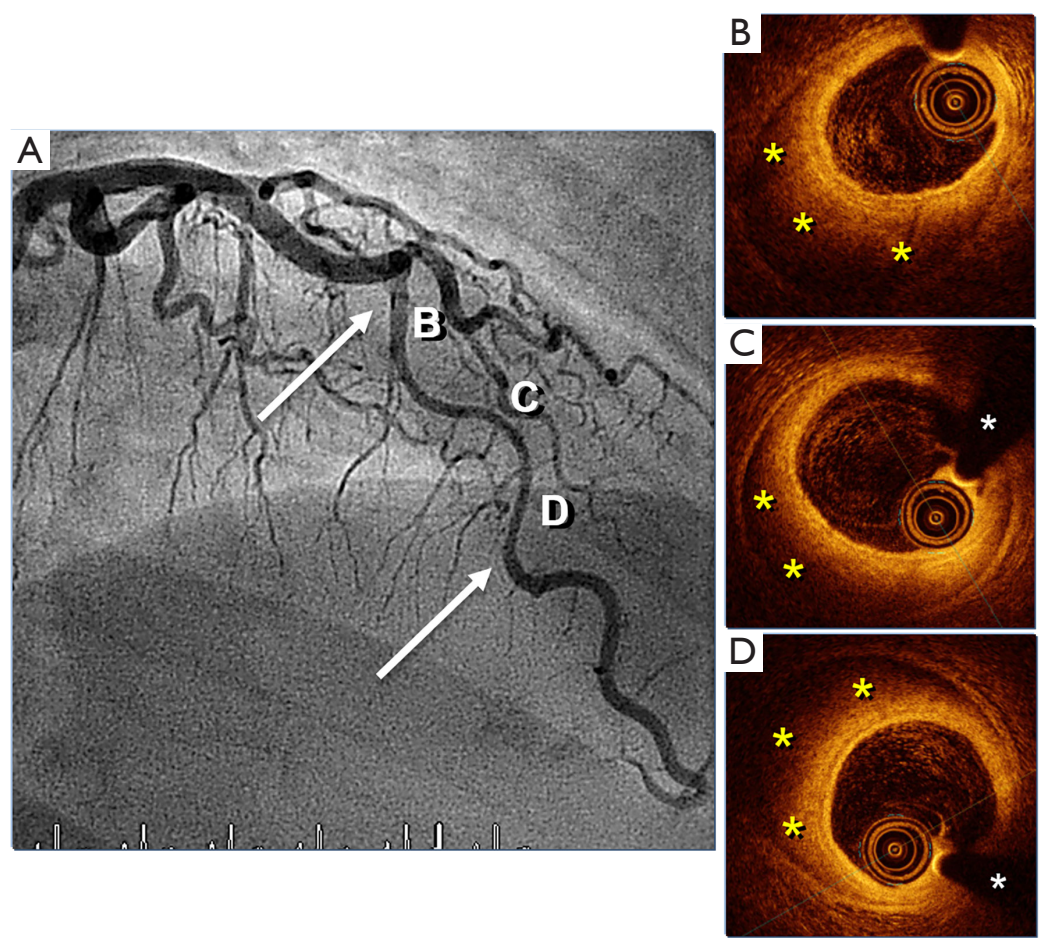

Figure 2 Young woman presenting with chest pain and anterior ST-segment changes on the ECG. (A) Coronary angiogram of a patient with SCAD demonstrating a diffuse narrowing (arrows) in the mid segment of a tortuous left anterior descending coronary artery. The image remained unchanged after the administration of intracoronary nitroglycerin; (B,C,D) optical coherence tomography images depicting a long intramural hematoma [yellow asterisk $\left(^{*}\right)$ ] with a relatively preserved coronary lumen. White asterisk $\left(^{*}\right)$ denotes wire artifact. SCAD, spontaneous coronary artery dissection.

appearance of the remaining coronary arteries). The use of the Saw classification (8) [type 1: double lumen with arterial staining; type 2: diffuse ( $>20 \mathrm{~mm}$ length) stenosis (2A, normal proximal and distal segments; $2 \mathrm{~B}$, diffuse narrowing extending to the distal tip); and type 3: focal stenosis mimicking ACAD], was proposed by the AHA statement. Nearly two-thirds of patients presents with a type 2 angiographic pattern (Figures 2 and 3).

Intracoronary imaging is frequently required to confirm the diagnosis in patients with intramural hematoma (9-11). Intravascular ultrasound provides a better penetration in the vessel wall and enables adequate visualization of the entire extent of the false lumen or intramural hematoma (11). Optical coherence tomography (OCT) has 10 times better spatial resolution but has a shallower penetration. This imaging technique is more accurate to detect the intimal tear and visualize the dissecting membrane $(9,10)$ (Figures 2 and 3). In patients with diffuse disease visualizing only the proximal segments of the affected vessel may be sufficient for an adequate diagnosis. Due to the underlying wall frailty coronary angiography, and especially intracoronary imaging, should be performed with major care to avoid disease propagation or iatrogenic dissections (1).

Cardiac CT imaging is frequently used in the "triple rule-out" protocols used for patients with chest pain. In the acute phase identification of SCAD with CT angiography is very challenging. However, this noninvasive technique may be used for follow-up purposes, particularly in patients with SCAD affecting proximal coronary segments (1).

Screening for associated conditions has been incorporated as part of the diagnostic work-up in SCAD patients $(1,6)$. Identification of FMD and other extracoronary vascular abnormalities is important because aneurysms and dissections in large arteries may require specific therapy. A complete vascular examination, including assessment of pulse asymmetry and abdominal bruits, should be performed in all patients with SCAD. Systematic imaging of several vascular territories is also recommended. Cerebral aneurysms can be detected in $10 \%$ to $20 \%$ of these patients (1). Catheter-based angiography is the best method to explore 


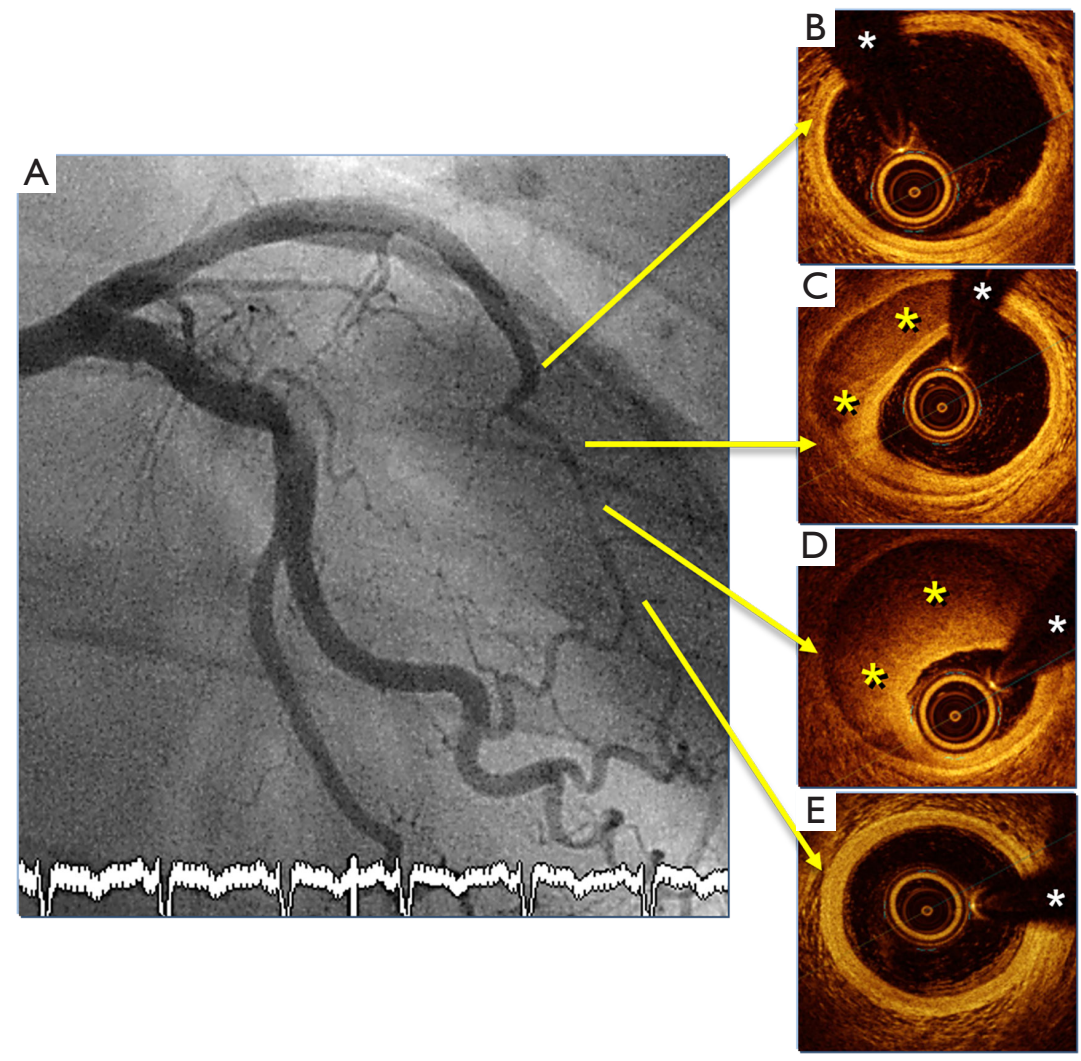

Figure 3 Patient presenting with ACS. (A) Coronary angiography of a patient with SCAD showing a relative focal lumen narrowing at the mid segment of the left anterior descending coronary artery; $(\mathrm{B}, \mathrm{C}, \mathrm{D}, \mathrm{E})$ optical coherence tomography images demonstrating a normal vessel wall at the edges of the stenosis $(\mathrm{B}, \mathrm{E})$ and a severe lumen compromised caused by an intramural hematoma [yellow asterisk $\left(^{*}\right)$ ] $(\mathrm{C}, \mathrm{D})$. White asterisk $\left(^{*}\right)$ denotes wire artifact. SCAD, spontaneous coronary artery dissection; ACS, acute coronary syndrome.

these vascular territories due to its high resolution. In selected cases this can be performed during diagnostic coronary angiography $(1,6)$. However, digital subtraction angiography is usually not required. CT angiography provides an excellent noninvasive means to screen for FMD and other arteriopathies as its resolution is higher than that of magnetic resonance imaging. However, magnetic resonance imaging does not require radiation or contrast agents and its resolution has been improved with the advent of $3.0 \mathrm{~T}$ technology. Exposure to ionizing radiation may be a cause of concern in young patients. Duplex ultrasonography has a much lower resolution and its use has been largely abandoned for this purpose (1).

Finally, SCAD is usually sporadic and familial association is rare. The yield of routine genetic testing in unselected SCAD patients is low and thus not recommended. However, genetic testing may be of value in selected patients with aortopathies or systemic connective tissue diseases (1).

\section{Clinical management}

An early invasive strategy is indicated for most patients with ACS. However, in contradistinction to patients with ACAD, a conservative management rather than revascularization is suggested for patients with $\operatorname{SCAD}(1,12)$. Observational data suggest that prognosis is favorable when patients with SCAD are managed conservatively (12). In addition, spontaneous healing of SCAD has been reported during follow-up in most cases (12). Nevertheless, revascularization should be always considered in patients with high-risk anatomy (i.e., left main), those hemodynamically unstable and those with ongoing ischemia $(1,12)$. In addition, urgent revascularization may be eventually required in up to $10 \%$ of patients selected for an early conservative strategy (1).

Coronary revascularization is very challenging in patients with $\operatorname{SCAD}(1,12,13)$. Observational studies have consistently suggested that coronary interventions in these 
patients are associated with poorer results and an increased risk of complications (13). Technical failure, including the presence of residual dissection, may occur in up to $50 \%$ of patients $(1,13)$. Angiographic success is higher when residual dissections or stenosis are not considered. However, emergency coronary artery bypass grafting may be required in $10 \%$ of patients undergoing coronary interventions (1). Extension of dissections and propagation of hematoma may lead to main vessel occlusion or loss of adjacent side-branches. SCAD frequently involves distal and small coronary segments that are difficult to treat with stents. Hematoma resorption may increase the risk of late malapposition and subsequent stent thrombosis. The femoral route may guarantee a more stable catheter sitting thus avoiding iatrogenic dissections (1). Several strategies for stent implantation have been suggested but comparative results are currently lacking. Fenestration, with or without subsequent stenting, has been suggested to allow decompression of the false lumen and enlargement of the true lumen (14). Finally, bioresorbable stents have been used in selected cases to provide temporal vascular scaffold and allow for complete vascular healing (14).

Limited evidence supports the value of coronary surgery in SCAD patients (13). This strategy is reserved for patients with left main or challenging multiple proximal dissections. Although coronary surgery provides acute success in most patients a high rate of both venous and arterial failure has been reported during follow-up $(1,13)$. Competitive flow resulting from healing of the native coronary vessel has been implicated. Unfortunately, coronary surgery does not provide protection against SCAD recurrences.

Prolonged in-hospital monitoring should be part of the conservative strategy. Repeat angiography should be considered in cases with ischemia recurrence although recurrent chest pain does not necessarily imply SCAD extension or recurrences. Indeed, chest pain is very frequent after the initial episode and differential diagnosis is very challenging (1). Evidence for other supportive therapies, including intra-aortic balloon pumping, left ventricular assist devices, extracorporeal membrane oxygenator, or implantable defibrillator, remains very limited (1). Risk for iatrogenic damage of large arteries should be considered especially in patients with associated FMD.

In the absence of clear targets and lack of randomized clinical trials, medical therapies suffer from major gaps in evidence (1). Therefore, current recommendations are largely based on expert opinions (1-4). Antithrombotic strategies remain controversial in SCAD patients and clearly differ from the aggressive strategies currently recommended for patients with ACS and ACAD (1). Risk of hematoma or dissection extension remains a theoretical concern. Some experts suggest discontinuation of anticoagulation after the diagnosis. Moreover, evidence on the value of dual antiplatelet therapy in patients not treated with coronary interventions, is lacking (1). Some experts suggest maintenance of dual antiplatelet therapy for 1 year. Data from some clinical practice registries suggest that this is the most frequently selected strategy (1). Other experts consider a limited (1-3 months) use of the dual antiplatelet therapy followed by long-term aspirin treatment. The risk for menorrhagia in premenopausal women should be taken into consideration and therapeutic decisions in this regard should be individualized (1).

Betablockers should be used in patients with left ventricular dysfunction, hypertension or arrhythmias. Most investigators also advocate the systematic use of these drugs to reduce coronary wall stress (1-4). However, betablockers may facilitate coronary vasospasm and this is of concern in some patients with SCAD. Observational data suggest that betablockers may reduce the risk of recurrences (15). Stains should be used in patients meeting criteria for primary prevention of ACAD. An observational study suggested that statin therapy might increase the risk of recurrences (16) but more recent reports failed to confirm this adverse association $(1,15)$. Nitrates and calcium channel blockers are widely used in patients with angina or chest pain.

Patients with SCAD are at risk for adverse events during follow-up and therefore a close clinical surveillance should be organized. Recurrences are the major cause of late adverse events $(15,16)$. During follow-up de novo dissections tend to occur in different coronary segments whereas in the acute phase extension of the index dissection is more common (1). Predictors of recurrences remain unclear although coronary tortuosity has been identified as a potential risk factor (17). Alternatively, FMD has not been associated with an increased risk for recurrences.

Management of pregnancy-related SCAD is particularly challenging. This problem is nicely addressed in the AHA scientific statement (1). A multidisciplinary approach is recommended for these patients. Overall, general recommendations are similar and conservative medical management is also preferred. Betablockers may cause low fetal growth and, in this scenario, labetalol is the preferred drug. Maternal and fetus status considerations become of upmost importance. Special attention should be also paid to pharmacological therapy, particularly in breastfeeding 
women. Atenolol should be avoided during breastfeeding (1).

Cardiac rehabilitation is also addressed in great detail in the AHA scientific statement (1). Current evidence suggests low referral of these patients to rehabilitation programs despite emerging evidence supporting its benefits in this particular setting $(18,19)$. Some physicians fear that the exercise prescribed in these programs could trigger recurrences. Health care provides should prescribe a prudent approach to exercise in these patients. Highintensity activities, strain, severe weight lifting and Valsalva maneuvers, should be avoided (1).

Counselling regarding pregnancy, contraception and hormone replacement therapy after SCAD is also discussed in depth in the AHA scientific statement (1). Observational evidence suggests a potential risk of repeat SCAD with pregnancy and also highlights the particularly poor prognosis of SCAD in this situation. Patients selecting to attempt pregnancy should be referred to experienced tertiary centers with multidisciplinary teams, including maternal-fetal specialist and obstetric anesthesiologists, for careful planning of pregnancy, labor and postpartum care. Epidural anesthesia, left lateral decubitus positioning and careful blood pressure monitoring is frequently advocated. Vaginal delivered is usually preferred but always trying to minimize maternal effort (1).

Oral contraceptives and postmenopausal hormonal therapy should be avoided when possible in patients with previous SCAD, but patient's preferences should be also considered and eventually decisions should be individualized. Likewise, abnormal uterine bleeding is frequent in premenopausal women on dual antiplatelet therapy. Indication of dual antiplatelet therapy should be reassessed in this scenario. Hormone-eluting intrauterine devices should be considered in patients with abnormal uterine bleeding and also to prevent pregnancy (1).

Physiological considerations are also very important. These young patients frequently develop anxiety or depression when faced with the uncertainties on their diagnosis, treatment and prognosis (20). Patient-based online communities and social media platforms provide an effective means of virtual peer support for these patients (1).

\section{Conclusions}

Well-designed, large, nation-wide, prospective studies are required to address major gaps in knowledge on this unique condition. The AHA scientific statement on SCAD clearly highlights major research areas and key questions regarding: (I) epidemiology, (II) pathogenesis, (III) diagnosis and (IV) treatment (1). Major efforts are required to spur collaboration research in order to allow advances in the diagnostic and management of this disease. The AHA and ESC scientific statements represents excellent tools to increase awareness and optimize management of patients with this elusive clinical entity $(1,2)$. However, additional evidence is urgently required to fill current gaps in knowledge and allow a paradigm shift, moving from expertbased recommendations to evidence-base medicine.

\section{Acknowledgements}

None.

\section{Footnote}

Conflicts of Interest: The authors have no conflicts of interest to declare.

\section{References}

1. Hayes SN, Kim ESH, Saw J, et al. Spontaneous Coronary Artery Dissection: Current State of the Science: A Scientific Statement From the American Heart Association. Circulation 2018;137:e523-57.

2. Adlam D, Alfonso F, Maas A, et al. European Society of Cardiology, acute cardiovascular care association, SCAD study group: a position paper on spontaneous coronary artery dissection. Eur Heart J 2018. [Epub ahead of print].

3. Alfonso F, Bastante T, García-Guimaraes M, et al. Spontaneous coronary artery dissection: new insights into diagnosis and treatment. Coron Artery Dis 2016;27:696-706.

4. Saw J, Mancini GBJ, Humphries KH. Contemporary Review on Spontaneous Coronary Artery Dissection. J Am Coll Cardiol 2016;68:297-312

5. Saw J, Aymong E, Mancini GB, et al. Nonatherosclerotic coronary artery disease in young women. Can J Cardiol 2014;30:814-9.

6. Saw J, Ricci D, Starovoytov A, et al. Spontaneous coronary artery dissection: prevalence of predisposing conditions including fibromuscular dysplasia in a tertiary center cohort. JACC Cardiovasc Interv 2013;6:44-52

7. Saw J, Bezerra H, Gornik HL, et al. Angiographic and Intracoronary Manifestations of Coronary Fibromuscular Dysplasia. Circulation 2016;133:1548-59.

8. Saw J. Coronary angiogram classification of spontaneous coronary artery dissection. Catheter Cardiovasc Interv 
2014;84:1115-22.

9. Alfonso F, Canales E, Aleong G. Spontaneous coronary artery dissection: diagnosis by optical coherence tomography. Eur Heart J 2009;30:385.

10. Alfonso F, Paulo M, Gonzalo N, et al. Diagnosis of spontaneous coronary artery dissection by optical coherence tomography. J Am Coll Cardiol 2012;59:1073-9.

11. Paulo M, Sandoval J, Lennie V, et al. Combined use of OCT and IVUS in spontaneous coronary artery dissection. JACC Cardiovasc Imaging 2013;6:830-2.

12. Alfonso F, Paulo M, Lennie V, et al. Spontaneous coronary artery dissection: long-term follow-up of a large series of patients prospectively managed with a "conservative" therapeutic strategy. JACC Cardiovasc Interv 2012;5:1062-70.

13. Tweet MS, Eleid MF, Best PJ, et al. Spontaneous coronary artery dissection: revascularization versus conservative therapy. Circ Cardiovasc Interv 2014;7:777-86.

14. García-Guimaraes M, Bastante T, Cuesta J, et al. Hybrid Percutaneous Treatment of Iatrogenic Coronary Artery Dissection Complicating a Spontaneous Coronary Artery
Dissection. EuroIntervention 2018. [Epub ahead of print].

15. Saw J, Humphries K, Aymong E, et al. Spontaneous Coronary Artery Dissection: Clinical Outcomes and Risk of Recurrence. J Am Coll Cardiol 2017;70:1148-58.

16. Tweet MS, Hayes SN, Pitta SR, et al. Clinical features, management, and prognosis of spontaneous coronary artery dissection. Circulation 2012;126:579-88.

17. Eleid MF, Guddeti RR, Tweet MS, et al. Coronary artery tortuosity in spontaneous coronary artery dissection: angiographic characteristics and clinical implications. Circ Cardiovasc Interv 2014;7:656-62.

18. Chou AY, Prakash R, Rajala J, et al. The First Dedicated Cardiac Rehabilitation Program for Patients With Spontaneous Coronary Artery Dissection: Description and Initial Results. Can J Cardiol 2016;32:554-60

19. Krittanawong C, Tweet MS, Hayes SE, et al. Usefulness of Cardiac Rehabilitation After Spontaneous Coronary Artery Dissection. Am J Cardiol 2016;117:1604-9.

20. Liang JJ, Tweet MS, Hayes SE, et al. Prevalence and predictors of depression and anxiety among survivors of myocardial infarction due to spontaneous coronary artery dissection. J Cardiopulm Rehabil Prev 2014;34:138-42.
Cite this article as: Alfonso F, García-Guimaraes M, Bastante T, de la Cuerda F, Antuña P, Cuesta J, Rivero F. Spontaneous coronary artery dissection: from expert consensus statements to evidence-based medicine. J Thorac Dis 2018;10(7):4602-4608. doi: $10.21037 /$ jtd.2018.07.10 